\title{
Receding Horizon Pseudospectral Optimal Control for Wave Energy Conversion
}

\author{
Romain Genest \\ COER, Electronic Egineering Department \\ NUIM \\ Maynooth, Ireland \\ Email: rgenest@eeng.nuim.ie
}

\author{
John Ringwood \\ COER, Electronic Egineering Department \\ NUIM \\ Maynooth, Ireland \\ Email: john.ringwood@eeng.nuim.ie
}

\begin{abstract}
The present study introduces a real-time control algorithm for applications involving energy maximisation and subject to technological limitations. Development of Wave energy converters constitutes an actual topic of research, in their designs and fluid-interactions, and particularly on their controllability. Immersed WECs are subject to fluid-interaction forces, generating unusual solicitations specific to hydrodynamic equation, such as radiation force or excitation forces.

Various control strategies were recently developed and more advance control algorithms are currently applied on WECS, such as Model Predictive Control [1], or Pseudospectral optimal control [2]. Such control strategies, capable of maximizing the energy production while insuring the respect of path constraints, are more realistic and applicable in real conditions involving technological limitation aspects.

This paper presents a receding horizon type control, based on pseudospectral approach in the control problem resolution. Dealing with irregular waves on a fixed control horizon, the presented control need to work with nonperiodic functions, implying the change of the basis functions involved in the description of the state and control variables, namely the halfrange Chebyshev Fourier functions.

Application of the receding horizon control is presented for a generic WEC, and compared with a standard MPC algorithm.
\end{abstract}

\section{INTRODUCTION}

In the wave energy field, various control strategies have been developed, such as latching control [3], declutching control [4], phase-control [5] and compared [6]. Energy maximisation remains the main objective of a control algorithm, but despite the development of advanced control strategies, handling technological limitations during operation, is a necessary control ability. The respect of path constraints arises in nowadays since the development of various WECs and the multiplicity of experimental testing, bring the wave energy field into the design of applicable and practical control algorithms. Model Predictive Control (MPC), introduced in the wave energy domain [1], allows an energy maximisation while ensuring the respect of path constraints, leading to an interesting and realistic approach for the control of WEC. More recently, pseudospactral optimal control was introduced in an energy maximisation problem for wave energy conversion [7], and recently improved in [8] allowing its application in real-time via a receding control horizon. The present study introduces the receding horizon pseudospectral control and compare its performance with a standard MPC algorithm.

\section{OPTIMAL CONTROL PROBLEM FORMULATION}

As one degree of freedom systems constitute generic case studies to implement advance control strategies, facilitating the understanding of the control performance, the wave energy converter (WEC) considered in this study is constrained to move in heave only. The studied floating point absorber with a spherical shape, is set into motion by the incoming wave field. A power takeoff (PTO) module is responsible for converting the mechanical energy of the WEC into electrical energy. The absorbed energy is potentially usable and connectible to a local grid.

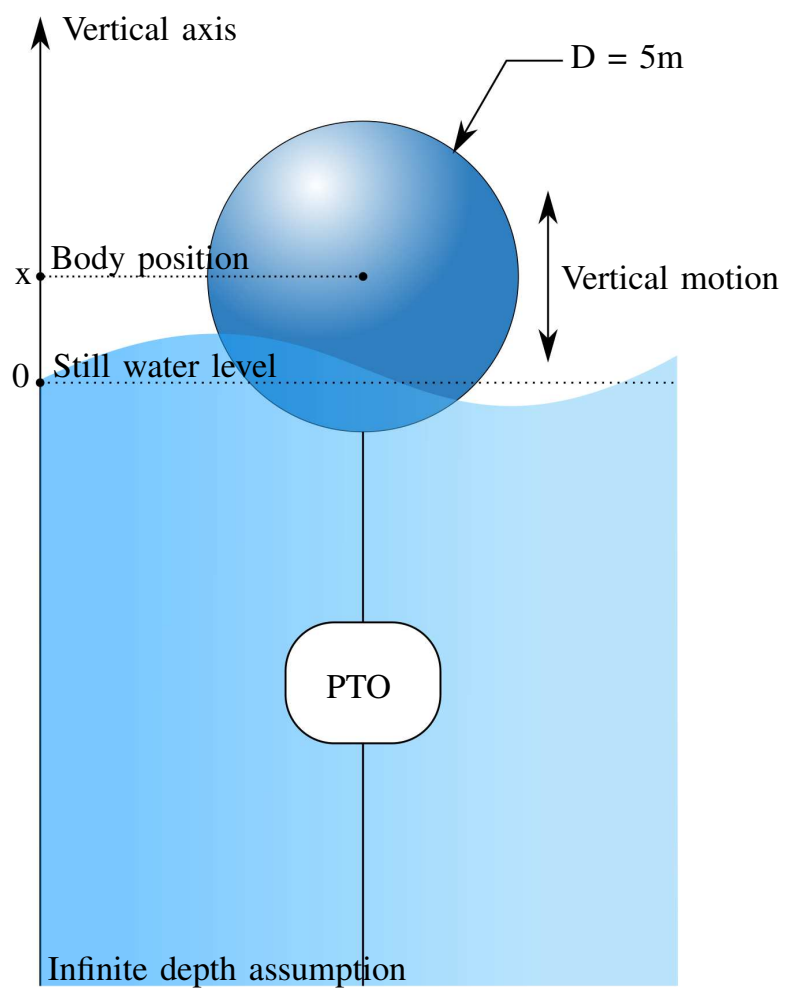

Fig. 1. Wave energy converter

The control algorithm, implemented on an embedded system, is crucial to command the actuators and more generally, the PTO parameters. Not only, the control algorithm maximises the WEC energy absorption, but it also ensures 
the respect of mechanical or electrical limitations. In our case study, the control algorithm is defined to generate an optimal trajectory followed in real-time by the WEC through the command of the PTO.

The linearised WEC equation of motion, or Cummins' equation [9], is presented in (1).

$$
(m+\mu) \frac{d v}{d t}+\int_{0}^{t} K(t-\tau) v(\tau) d \tau+S_{H} x=F_{e x}+u
$$

where $x$ and $v$ represent the vertical position and the velocity of the system, $m$ and $\mu$ are, respectively, the mass and the infinite frequency added mass asymptote. $K$ is the kernel function of the radiation force, $S_{H}$ is the linearised hydrodynamic stiffness, $F_{e x}$ is the excitation force, corresponding to the pressure force generated by the incoming wave field and finally, and $u$ is the control force, i.e. the force applied by the PTO on the WEC's hull.

The absorbed energy over an give time horizon $T$, under the assumption of no energy losses, is given by,

$$
E=-\int_{0}^{T} u(t) v(t) d t
$$

The implemented control is design to maximize the energy absorption $E$ over a given control horizon $T$. One needs to exhibit motion and control trajectories to maximize the quantity $E$. In order to estimate the energy production, the excitation force is assumed to be known over the control horizon $T$ in the future. An estimation of the forthcoming values of $F_{e x}$ are estimated by a specific prediction algorithm based on wave elevation probes, and is not considered in this study.

Not all trajectories are acceptable in real conditions, course and control force limitations are obviously part of the control objectives and cannot be eluded without generating unrealistic trajectories. Path constraints are expressed in terms of inequality constraints over the whole control horizon $T$, and for $t \in[0, T]$,

$$
\begin{aligned}
& |x(t)| \leq X_{\max } \\
& |v(t)| \leq V_{\max } \\
& |u(t)| \leq U_{\max }
\end{aligned}
$$

where $X_{\max }, V_{\max }, U_{\max } \in \mathbb{R}^{+}$correspond to technological limitations. The control problem is fully defined, intending to exhibit motion and control trajectories that maximized the energy production defined in (2), while respecting the path constraints described in (5) and insuring realistic description of the system dynamics presented in (1).

\section{PSEUdOSPECTRAL OPTIMAL CONTROL}

The control problem defined in Section II falls into standard optimal control problematics, solved for various applications and for many years. Various methodologies were developed in order to answer optimal control problem, among which MPC and pseudospectral control. The algorithm presented here is based on the pseudospectral optimal control to generate the reference trajectory to be followed by the WEC [7].

Spectral methods are characterised by the projection of the state and control variables into a specific orthogonal set of basis functions as stated in (6).

$$
x(t) \approx x_{N}(t)=\sum_{i=1}^{N} a_{i} \psi_{i}(t)=\Psi \bar{x}
$$

where $\Psi=\left[\psi_{1}, \ldots, \psi_{N}\right]$. The optimal control is thusly expressed in terms of projection vectors derived from the trajectory projections, $\bar{x}=\left[a_{1}, \ldots, a_{N}\right]$, leading to a finiteorder control problem describing continuous control and state variables.

The choice of a particular set of basis functions is thighly related to the type of control problem considered and particularly on the type of control and state variables involved in the system dynamics. In the specific case of wave energy conversion, a legitimate choice for the basis functions are the Fourier function, i.e. orthogonal trigonometric functions. Approaches based on generalised truncated Fourier series is presented in for periodic control problems. One drawback of this methodology relies in the apparition of Gibbs phenomenon for nonperiodic control problem, i.e. control problem with different initial and final boundary conditions. The present study is based on a recent development on the Fourier extension of nonperiodic functions.

The orthogonal set of basis functions employed for the pseudospectral optimal control approach is based on half-range Chebyshev Fourier functions [10], defined from trigonometric polynomials called half-range Chebyshev polynomials $T_{k}$ and $U_{k}$ respectively of the first and second kind.

Definition 1. Let $T_{k}^{h}(y)$ be the unique normalized sequence of orthogonal polynomials satisfying

$$
\begin{aligned}
& \int_{0}^{1} T_{k}^{h}(y) y^{l} \frac{1}{\sqrt{1-y^{2}}} d y=0, \quad l=0, \ldots, k-1 \\
& \frac{4}{\pi} \int_{0}^{1} T_{k}^{h}(y)^{2} \frac{1}{\sqrt{1-y^{2}}} d y=1
\end{aligned}
$$

The set $\left\{T_{k}^{h}(y)\right\}_{k=0}^{\infty}$ is a set of half-range Chebyshev polynomials of the first kind.

Definition 2. Let $U_{k}^{h}(y)$ be the unique normalized sequence of orthogonal polynomials satisfying

$$
\begin{aligned}
& \int_{0}^{1} U_{k}^{h}(y) y^{l} \sqrt{1-y^{2}} d y=0, \quad l=0, \ldots, k-1 \\
& \frac{4}{\pi} \int_{0}^{1} U_{k}^{h}(y)^{2} \sqrt{1-y^{2}} d y=1
\end{aligned}
$$

The set $\left\{U_{k}^{h}(y)\right\}_{k=0}^{\infty}$ is a set of half-range Chebyshev polynomials of the second kind.

The set of orthogonal basis functions $\left\{\Psi_{i}(t)\right\}_{i=1}^{N}$ is defined by the union of the two sets $\left\{T_{k}\left(\cos \frac{\pi t}{2}\right)\right\}_{k=0}^{n}$ and $\left\{U_{k}\left(\cos \frac{\pi t}{2}\right) \sin \frac{\pi t}{2}\right\}_{k=0}^{n-1}$ called half-range Chebyshev Fourier functions of the first and second kind respectively. Their 
trigonometric definitions are particularly suited to approximate wave elevation or excitation forces involved in a wave energy maximisation problem, allowing fast convergent rate during the state and control variables projections, and bringing insights in the understanding of the phenomenon in the frequency domain since the HRCF functions are hierarchically organised by fundamental frequency.

Cost function, path constraints and dynamical constraints are expressed in terms of projection vectors reducing the computational time involved in solving the parametrised optimal control problem. Pseudospectral method considers the equality and inequality constraints at particular instants $t_{k}$, called collocation points, at which the constraints must be satisfied. Each constraints is expressed as a residual terms $R$ that needs to be minimized by the employed optimization algorithm. As an example, the dynamical equation of the WEC is described in terms of residuals at the collocation nodes in (11).

$$
R\left(t_{k}\right)=\Psi\left(t_{k}\right)\left((m+\mu) D \bar{v}+P \bar{v}+S_{H} \bar{x}-\bar{F}_{e x}-\bar{u}\right)=0
$$

where $D$ represents the differentiation matrix [11], and $P$ the convolution matrix defined in [8]. In the same way, the energy absorption, i.e. the cost function $J$ to maximize, is rewritten in the following form,

$$
J=-\bar{u}^{T} \bar{v}
$$

where ${ }^{T}$ denotes the transpose vector.

The obtained control problem designed to maximized the cost function (12), while insuring the nullity of the residuals at each collocation points $t_{k}$, such as (11) and under inequality constraints, can be solved using standard quadratic problem solving algorithms, such as active-set or interior-point methods. The obtained solution of the derived control problem will determined each projection vectors of the state and control variables. From the solution projections, the optimal trajectories can be computed in the time domain and used as a reference trajectory that the WEC needs to follow. For more information about the implementation of the half-range Chebyshev Fourier basis function, the reader may refer to the following article [15].

\section{RECEDING HORIZON CONTROL}

The control algorithm presented in this study is hierarchically organised into two nested loops responsible for, respectively, the generation and tracking of the optimal trajectory. The trajectory generation is done using the pseudospectral approach presented in the previous Section II. A standard backstepping method is employed in order to track the reference trajectory computed by the higher algorithm loop. The control will determine a new trajectory at each time-step of the trajectory generation loop, leading to a receding horizon control. The complete structure of the receding horizon control is presented in Figure 2.

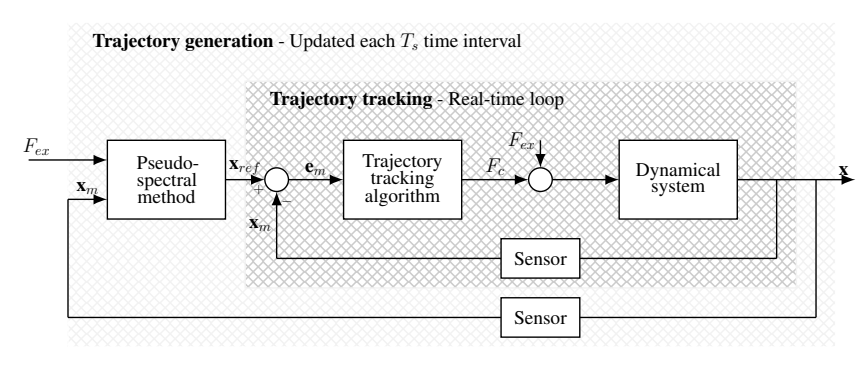

Fig. 2. Block diagram of the control algorithm

The time intervals between two consecutive calculations of the optimal trajectory is set to $T_{s}=0.5 s$. The trajectory tracking is implemented inside a Runge-Kutta temporal discretization with a time-step of $1 \mathrm{~ms}$. The backstepping control employed to track the optimal trajectory uses the second order ordinary differential equation (1) to define the control force command at each time-step of the Runge-Kutta simulation.

\section{MPC COMPARISONS}

The presented algorithm is tested in regular and irregular waves, and compared with a standard MPC algorithm presented in [12]. The full algorithm description is presented in [12] and follow a standard MPC formulation where the dynamical equation (1) is represented by a continuous time state-space. Different methods can be employed to define a state-space model for the convolution product in (1). In this study, two methodologies are studied, namely the Prony's method [13] and the NTNU Toolbox [14]. The Prony's method expressed the kernel of the radiation convolution product as a sum of complex exponential functions. The approximation obtained from the kernel function $K$ is used to define a first order differential equation and thus a continuous state-space. The NTNU Toolbox is based on a least square approximation of the Fourier transform of the kernel function by a fractional transfer function. The approximation based on the fractional transfer function is used to design a continuous time statespace. Using a First order holder, instead of a more common zero order holder,

$$
\begin{aligned}
x_{d}[k+1]= & \Phi(\Delta t) x_{d}[k] \\
& \cdots+\int_{k \Delta t}^{(k+1) \Delta t} \Phi((k+1) \Delta t-\tau) B u(\tau) d \tau \\
& \cdots+\int_{k \Delta t}^{(k+1) \Delta t} \Phi((k+1) \Delta t-\tau) B F_{e x}(\tau) d \tau
\end{aligned}
$$

with, $\Phi(t)=e^{A t}$ where $A$ is the matrix involved in the continuous time state-space dynamical model of the wave energy device described in equation (14).

$$
\dot{x}=A x+B\left(F_{e x}+u\right)
$$

Then it leads to a discrete-time state-space model on the form, 
TABLE I

PARAMETERS OF THE CONTROL ALGORITHMS

\begin{tabular}{|l|l|l|}
\hline Algorithm & MPC & RHPSC \\
\hline Control horizon $[\mathrm{s}]$ & 15 & 20 \\
Parameters & $\Delta t=0.1 \mathrm{~s}$ & $\mathrm{n}=10$ \\
\hline
\end{tabular}

$$
\begin{aligned}
x_{d}[k+1]= & \Phi(\Delta t) x_{d}[k] \\
& \cdots+\Gamma\left(u[k]+F_{e x}[k]\right) \\
& \cdots+\Lambda\left(\Delta u[k+1]+\Delta F_{e x}[k+1]\right)
\end{aligned}
$$

where,

$$
\begin{aligned}
\Delta u[k+1] & =u[k+1]-u[k] \\
\Delta F_{e x}[k+1] & =F_{e x}[k+1]-F_{e x}[k]
\end{aligned}
$$

and,

$$
\begin{aligned}
\Gamma & =A^{-1}(\Phi(\Delta t)-I) B \\
\Lambda & =A^{-1}(\Gamma-\Delta t B) / \Delta t
\end{aligned}
$$

For more information about the MPC implementation, the reader may refer to the following articles [12] and [15]. The cost function, representing the energy absorption over a fixed control horizon $T$ is expressed in the discrete-time based on the state-space defined in (16). As presented in [12], it leads to a quadratic cost function. The control problem is then solved using standard quadratic optimization algorithms.

The parameters used to implement both the receding horizon pseudospectral control and MPC are presented in Table I.

The control horizon, i.e. the time under which the control algorithm will maximised the energy production, is fixed to $15 s$ for MPC and $20 s$ for the pseudospectral approach. A full convergence study, showing the relative performance of MPS and RHPSC, can be found in [15]. This convergence study was performed to derive a fair comparison between MPC and PS control where both of the algorithms were tuned to give the best trade-off between accuracy and computational time. The time-step employed to run the MPC algorithm is thus fixed to $0.1 \mathrm{~s}$. The number of basis functions involved in the description of the control and state variables in the pseudospectral method is fixed to 21 .

The computational time comparison uniquely involves the resolution of the derived QP for MPC and Pseudospectral control. Both algorithms need additional computational time either to interpolate the optimal control force at each time step of the real-time loop for MPC, or to estimate the control force, based on a backstepping approach, for a PS contol. In both cases, the additional computational time is negligeable compared to the time needed for the resolution of the QP.

Not all recent control strategies are compared in the present paper, especially [16]; since MPC and PS present directly competing formulations, describing state and control variables with a particular sets of basis functions and leading to a constrained QP optimisation, their direct comparison seems natural.

\section{A. Regular waves}

With the parameters defined by Table I, the MPC and receding horizon pseudospectral control were tested under regular waves solicitations for period varying between $5 s$ and 15s. Quadratic optimization problem are solved, for both MPC and receding horizon pseudospectral control, with a standard active-set method from the quadrprog function of MATLAB.

The energy absorption presented in Figure 3, for regular incoming waves under control without path constraints, is normalized by the theoretical maximum derived from the optimal complex-conjugate control [17]. The computational time presented corresponds to the time needed to solve each quadratic problem with am active-set methodology. Both MPC and the pseudospectral control present the same energy capture between $85 \%$ and $95 \%$ of the theoretical maximum. For both control algorithms, the absorbed energy is decreasing for large regular wave periods, since the control horizon is fixed, smaller wave periods allows a larger number of waves taken into account within the control horizon and thus a greater accuracy. The computation time does not depend on the incoming wave periods, but is shown to be around three times smaller for the pseudospectral control, around $5 \mathrm{~ms}$, than for the standard MPC, with a computational time of $15 \mathrm{~ms}$.
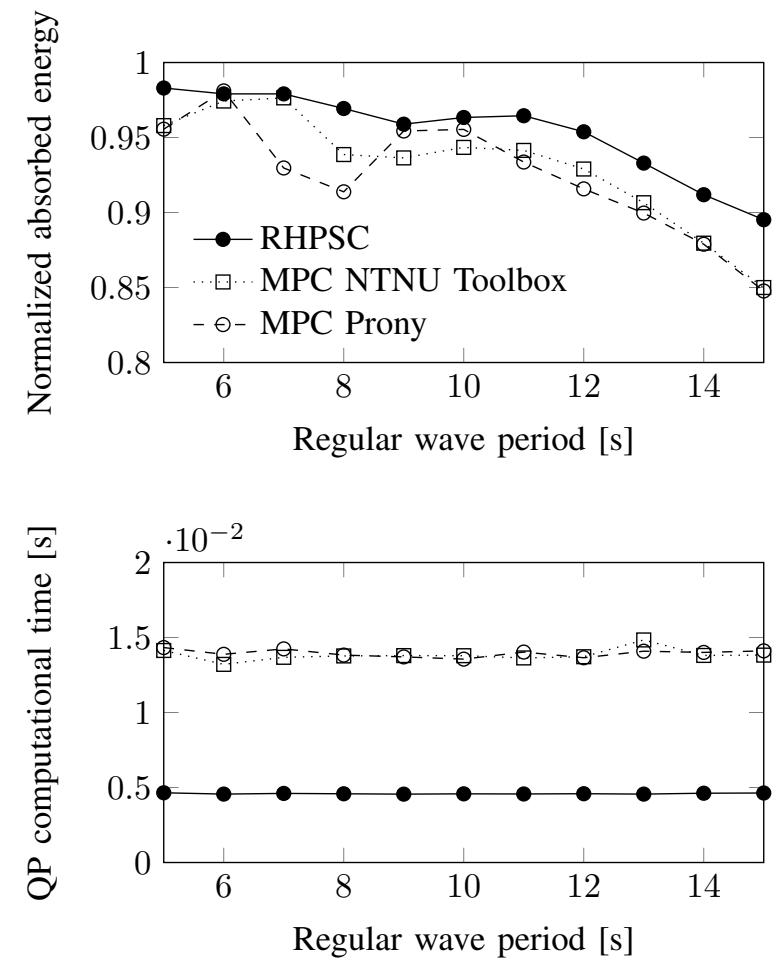

Fig. 3. Normalised absorbed power and QP computational time for MPC and RHPSC algorithm for different regular wave periods.

Figure 4 presents the normalised energy absorption and the computational time needed for both MPC and the pseudospectral control, but with path constraints, i.e. limitation on the 
position and velocity of the device. The WEC position is limited between $\pm 0.1 \mathrm{~m}$ and its velocity between $\pm 0.1 \mathrm{~m} / \mathrm{s}$. The impact of such technical limitations is significant, and increase with the wave period of the waves. Indeed, larger wave periods, generate greater excitation forces and increase the body motion amplitudes, since the constraints are kept constant for all the different regular waves tested, their impact will be higher for larger wave periods. No conclusion can be made on the actual amount of absorbed energy, since the results show only a normalised energy production. MPC and pseudospectral control are affected in the same way by technological limitations, and provide approximately the same amount of absorbed energy. However, while comparing the computational times involved in the resolution of each quadratic problem, the presence of path constraints are much more penalising for MPC than for the pseudospectral approach. Going from $15 \mathrm{~ms}$ to $100 \mathrm{~ms}$ for the MPC algorithm, it only goes from $5 \mathrm{~ms}$ to $20 \mathrm{~ms}$ for the pseudospectral approach. This might be due to the smaller number of variables involved in the quadratic problem resolution.
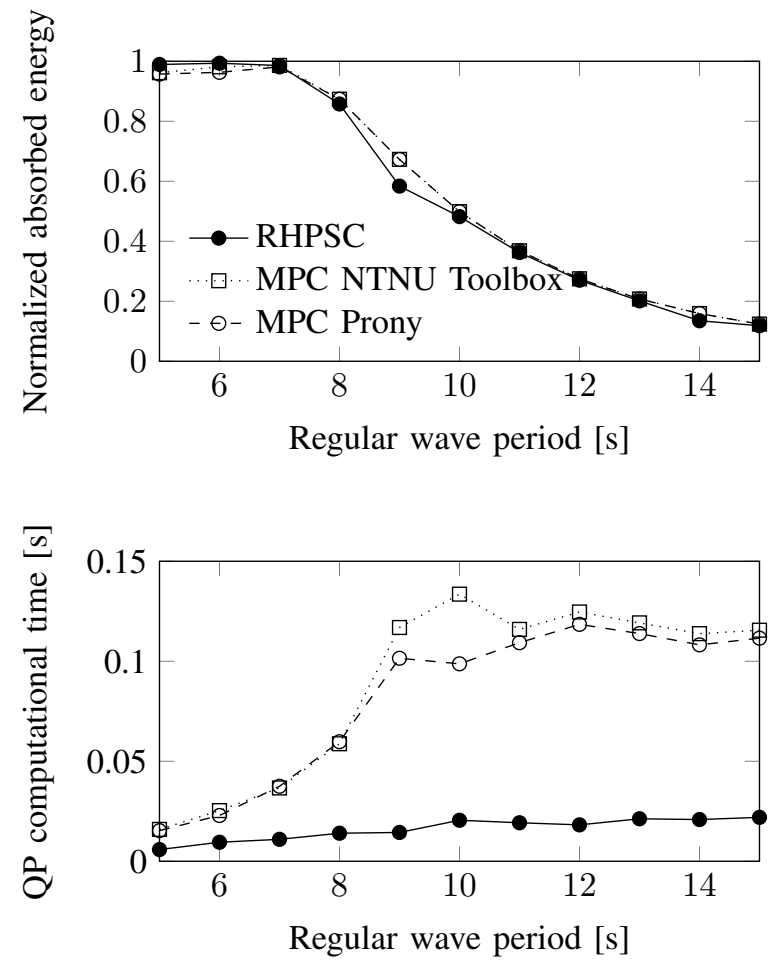

Fig. 4. Normalised absorbed power and QP computation time for MPC and RHPSC algorithms for different regular wave periods under position and velocity path constraints.

\section{B. Irregular waves}

Irregular waves generated from a Pierson-Moskowitz spectrum discretized in frequency between $0.02 \mathrm{~Hz}$ and $0.5 \mathrm{~Hz}$ with a frequency step of $d f=5 \cdot 10^{-3} \mathrm{~Hz}$, are used to immersed the WEC under control in a more realistic sea. Normalised energy absorption and computational time are presented in Figure 5. The irregular aspect of the excitation force does not affect significantly neither the energy absorption neither the computational time. As in the regular waves study, the energy absorption diminution with large wave period is significant. Since in a $15 \mathrm{~s}$ peak period spectrum, much larger wave period are generated, the energy drop is more significant for irregular waves, and normalise energy absorption is found between $90 \%$ and $95 \%$ for the pseudospectral approach and between $80 \%$ and $95 \%$ for MPC.
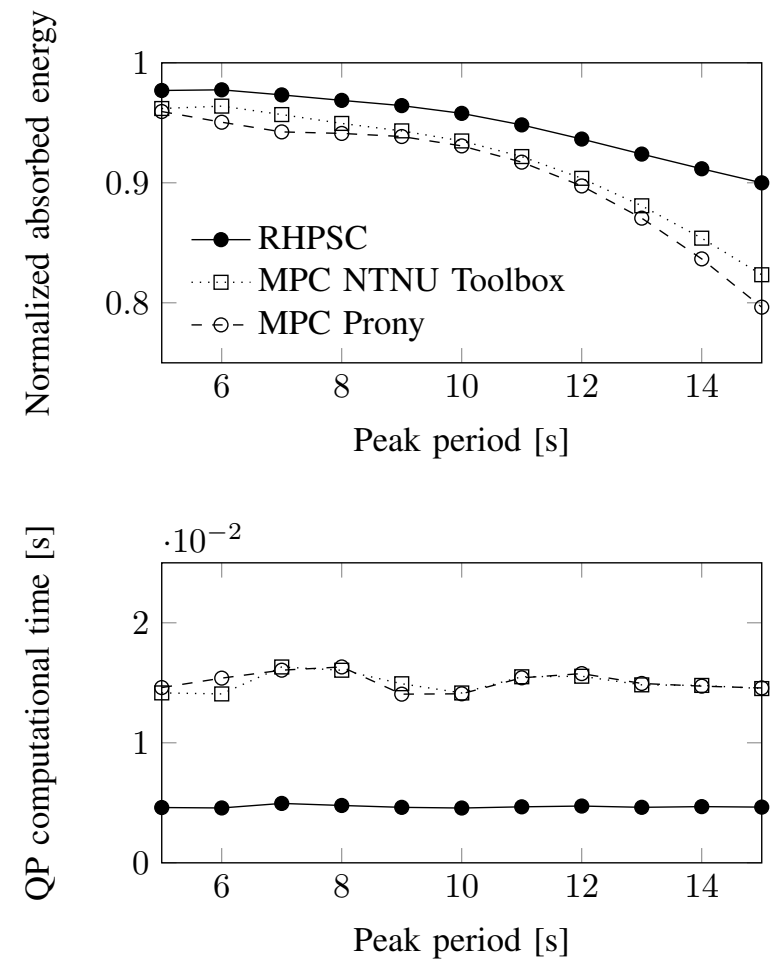

Fig. 5. Normalised absorbed power and QP computational time for MPC and RHPSC algorithm for different irregular sea states.

Time series of the position, velocity and control force obtained from WEC controlled with both MPC and pseudospectral control are presented in Figure 6 without any path constraints. All the proposed trajectories follow the optimal one determined from the complex-conjugate control [17].

To illustrate the ability of both control algorithms to handle path constraints, i.e. technological limitations, time series of the position, velocity and control force are presented in Figure 7 for a controlled WEC under path constraints. Both controls respect the imposed technological limitations, while absorbing maximum energy from the waves. The presented trajectories for MPC and pseudospectral control are almost identical, and lead to the same amount of absorbed energy.

\section{CONCLUSION}

Control strategies presented in this study, maximise the energy production of a particular WEC and ensure the respect of path constraints, representing technological limitations such as limited stroke or control force. MPC and pseudospectral 

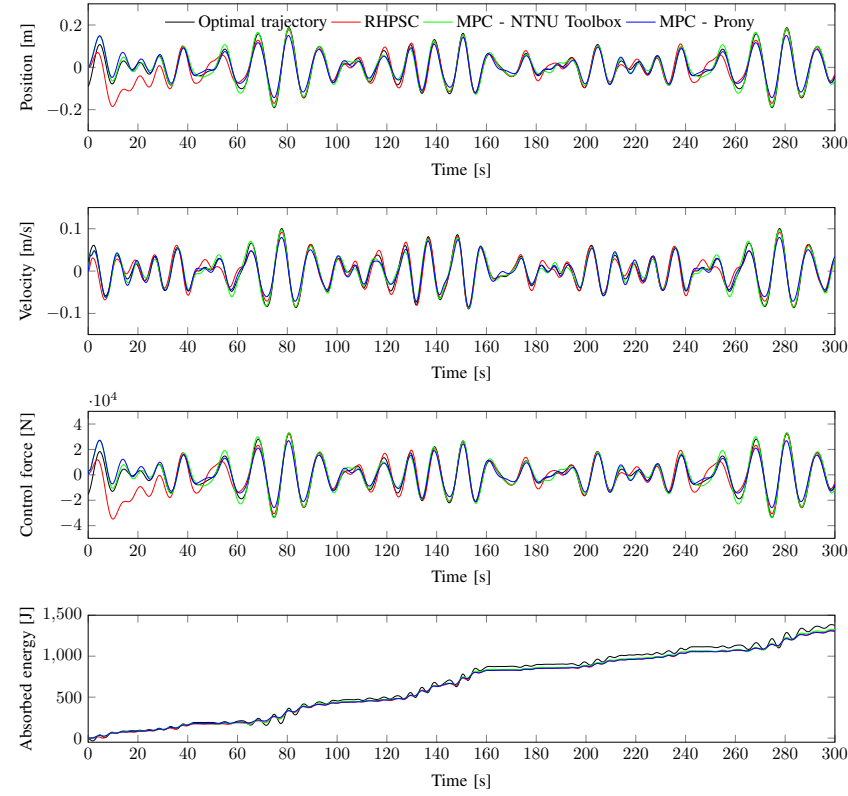

Fig. 6. Normalised absorbed power and QP computational time for MPC and RHPSC algorithms for different irregular sea states.
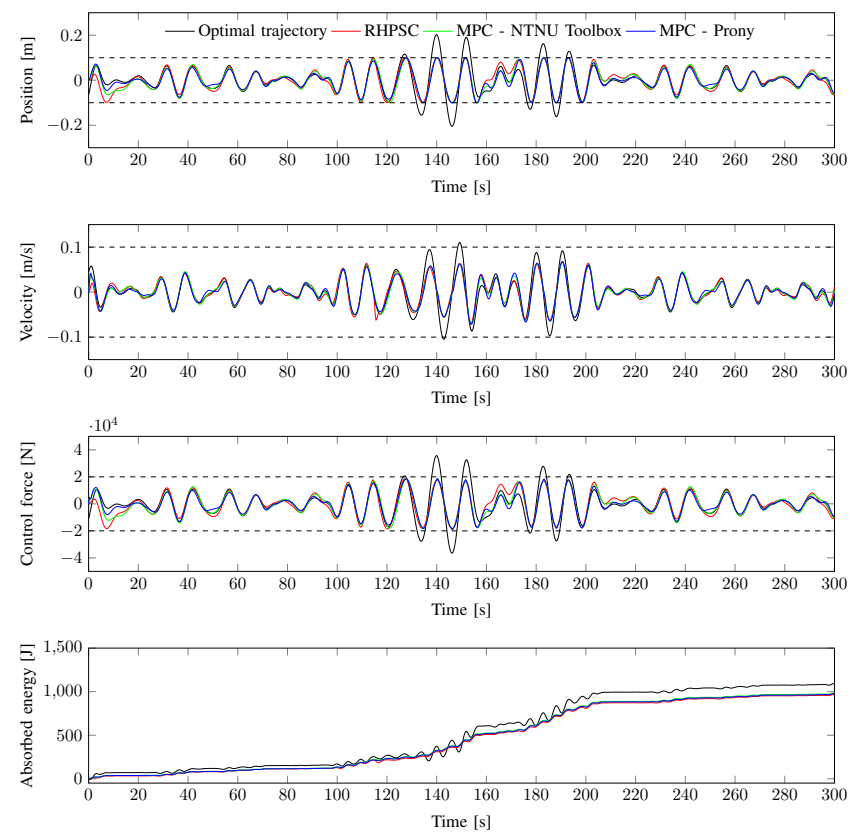

Fig. 7. Normalised absorbed power and QP computational time for MPC and RHPSC algorithms for different irregular sea states under path constraints.

control algorithms contribute to deliver to wave energy investors, a more advance, realistic and practical control strategy. Both of the algorithms tested in the present study, and based on a linear dynamical model of the considered WEC, can be run in real-time.

Further improvements will be needed to design an efficient control for WEC, since the fluid-interaction forces involved in its equation of motion remains highly nonlinear. The fluid forces acting on the WEC's hull depend on the immersed surface that is changing over time, depending on both the wave elevation and the body position. Additional nonlinear viscous forces need to be considered, since large velocity magnitude generate a higher drag force and turbulence effects could arise around sharp hull edges. Finally, the efficiency of the PTO, and its chosen technology, i.e. electrical or hydraulic PTO, affects the way the WEC needs to be controlled. The listed nonlinear effects, lead to a control strategy that will have to handle nonlinear dynamical equations, nonlinear cost function and nonlinear path constraints, in a effective way and a limited computational time.

\section{ACKNOWLEDGMENT}

This material is based upon works supported by Science Foundation Ireland under Grant No. 13/IA/1886.

\section{REFERENCES}

[1] J. Hals, J. Falnes, and T. Moan, "Constrained optimal control of a heaving buoy wave-energy converter," Journal of Offshore Mechanics and Arctic Engineering, vol. 133, no. 1, p. 011401, 2011.

[2] G. Bacelli and J. Ringwood, "Constrained control of arrays of wave energy devices," International Journal of Marine Energy, vol. 34, pp. e53 - e69, 2013, special Issue Selected Papers - \{EWTEC2013\}.

[3] A. Babarit and A. Clément, "Optimal latching control of a wave energy device in regular and irregular waves," Applied Ocean Research, vol. 28, no. 2, pp. 77-91, 2006.

[4] A. Babarit, M. Guglielmi, and A. H. Clément, "Declutching control of a wave energy converter," Ocean Engineering, vol. 36, no. 12, pp. 10151024, 2009

[5] P. R. Costa, P. B. Garcia-Rosa, and S. F. Estefen, "Phase control strategy for a wave energy hyperbaric converter," Ocean Engineering, vol. 37, no. 17 , pp. 1483-1490, 2010.

[6] J. Hals, J. Falnes, and T. Moan, "A comparison of selected strategies for adaptive control of wave energy converters," Journal of Offshore Mechanics and Arctic Engineering, vol. 133, no. 3, p. 031101, 2011.

[7] G. Bacelli and J. Ringwood, "Constrained control of arrays of wave energy devices," International Journal of Marine Energy, vol. 3, no. 4 pp. e53-e69, 2013.

[8] R. Genest and J. V. Ringwood, "Receding horizon pseudospectral control for energy maximization with application to wave energy devices," IEEE Transactions on Control Systems Technology, no. 99, pp. 1-10, 2016.

[9] W. Cummins, "The impulse response function and ship motions," DTIC Document, Tech. Rep., 1962.

[10] D. Huybrechs, "On the Fourier extension of nonperiodic functions," SIAM Journal on Numerical Analysis, vol. 47, no. 6, pp. 4326-4355, 2010.

[11] B. Orel and A. Perne, "Computations with half-range chebyshev polynomials," Journal of Computational and Applied Mathematics, vol. 236, no. 7 , pp. 1753-1765, 2012.

[12] J. A. Cretel, G. Lightbody, G. P. Thomas, and A. W. Lewis, "Maximisation of energy capture by a wave-energy point absorber using model predictive control," in Proceedings of the 18th IFAC World Congress, Milano, Italy, Aug, 2011, pp. 3714-3721.

[13] G. Duclos, A. H. Clément, and G. Chatry, "Absorption of outgoing waves in a numerical wave tank using a self-adaptive boundary condition," International Journal of Offshore and Polar Engineering, vol. 11, no. 03, 2001.

[14] T. Perez and T. I. Fossen, "A MATLAB toolbox for parametric identification of radiation-force models of ships and offshore structures," 2009.

[15] R. Genest and J. V. Ringwood, "A critical comparison of modelpredictive and pseudospectral control for wave energy devices," Journal of Ocean Engineering and Marine Energy, pp. 1-15, 2016.

[16] E. Abraham and E. C. Kerrigan, "Optimal active control and optimization of a wave energy converter," Sustainable Energy, IEEE Transactions on, vol. 4, no. 2, pp. 324-332, 2013.

[17] J. Falnes, Ocean waves and oscillating systems: linear interactions including wave-energy extraction. Cambridge University Press, 2002. 